\title{
A superficial early colitic cancer that resembled a laterally spreading tumor on chromoendoscopy
}

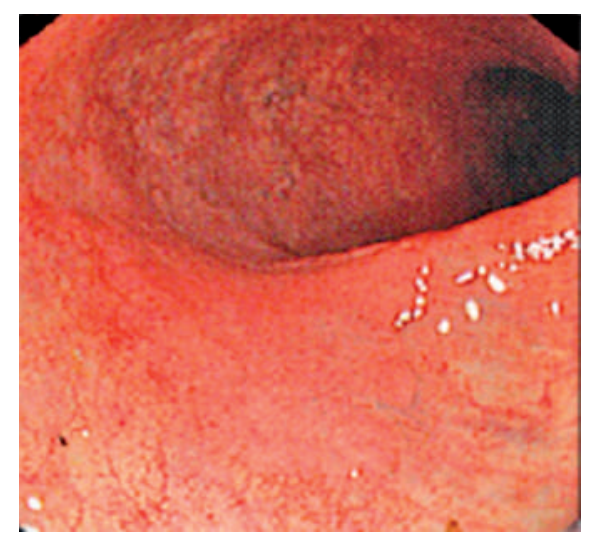

Figure 1 Colonoscopic view of the flat, reddish lesion that was observed in the sigmoid colon. The margin of the lesion could not be clearly visualized.

Patients with longstanding and extensive ulcerative colitis may be entered into a surveillance program [1], but the detection of ulcerative colitis-related colorectal neoplasia ("colitic cancer") during conventional colonoscopy is difficult. Because of this, chromoendoscopy [2] and magnifying chromoendoscopy [3] have recently been proposed as adjuvant techniques for the detection of this type of colorectal neoplasia.

A 35-year-old man with a 21-year history of extensive ulcerative colitis underwent a surveillance colonoscopy. A flat, reddish lesion was detected in the sigmoid colon (Figure 1). After the application of indigo carmine dye, the margin of the lesion could be clearly visualized and the lesion appeared to be a flat adenoma with a broad base, resembling a laterally spreading tumor (Figure 2). Magnifying chromoendoscopy showed a "noninvasive" pit pattern [4], suggesting that this was an intramucosal neoplastic lesion (Figure 3 ). It was therefore decided to treat this lesion and the surrounding normal-looking mucosa (which had a type I pit pattern) by endoscopic submucosal dissection [5]. Histopathological examination of the resected specimen showed a well-differentiated adenocarcinoma with submucosal and lymphatic invasion; the depth of invasion was $500 \mu \mathrm{m}$ from the muscularis

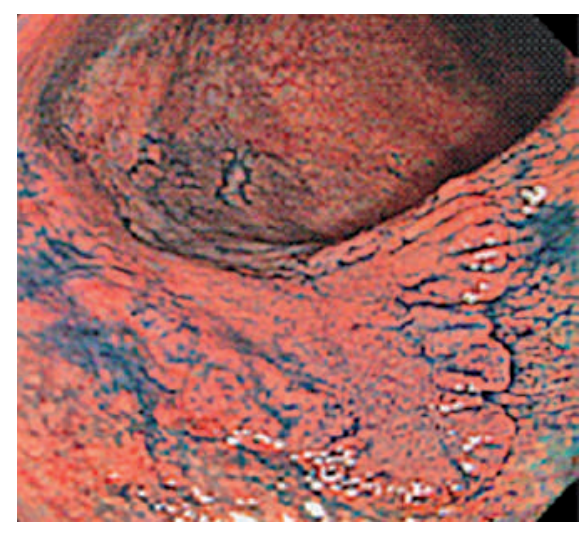

Figure 2 The margin of the lesion became much clearer after the application of indigo carmine dye.

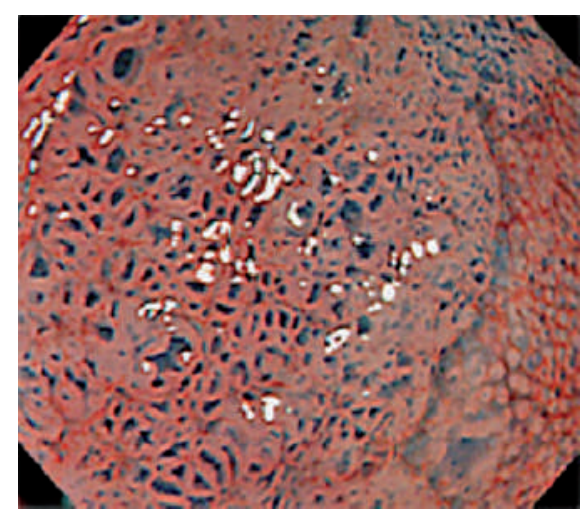

Figure 3 Magnifying chromoendoscopy revealed a noninvasive pit pattern, similar to a type IIIL pit pattern. The lesion was treated by endoscopic submucosal dissection (ESD).

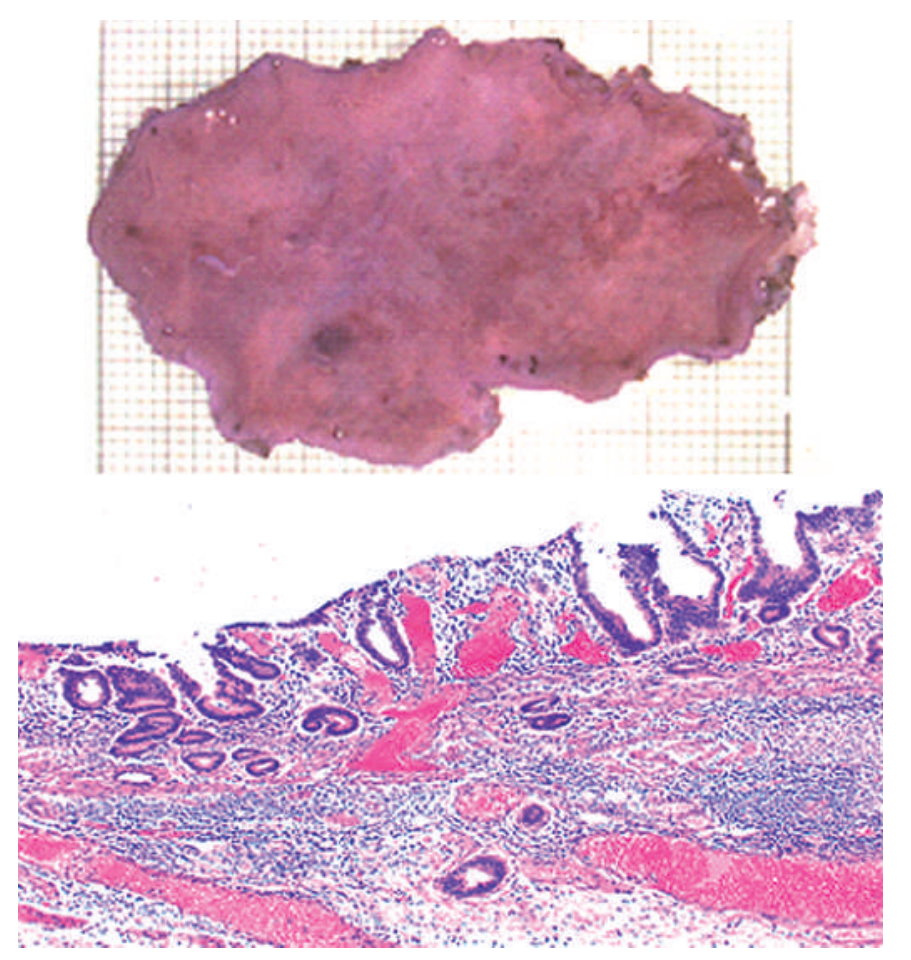

Figure 4 Histopathological examination of the resected specimen confirmed the lesion to be a well-differentiated adenocarcinoma with submucosal and lymphatic invasion. The depth of invasion was $500 \mu \mathrm{m}$ from the muscularis mucosa (hematoxylin \& eosin stain).

mucosa (Figure 4). Immunostaining for p53 was diffusely positive in most of the lesion and the adjacent mucosa also showed high-grade dysplasia. On the basis of these histopathological findings, this lesion was diagnosed as a colitic cancer, and a total colectomy was performed. Histopathological examination of the mucosa surrounding the scar after endoscopic submucosal dissection revealed high-grade dysplasia (Figure 5, 6).
This colitic cancer was diagnosed at an early stage by chromoendoscopy and magnifying chromoendoscopy, but the extent of tumor invasion was misdiagnosed. Histopathologically, the structure of the glands, especially the density of the glands of the neoplastic mucosa adjacent to the main lesion, resembled that of

DOI: $10.1055 / s-2006-944868$ 


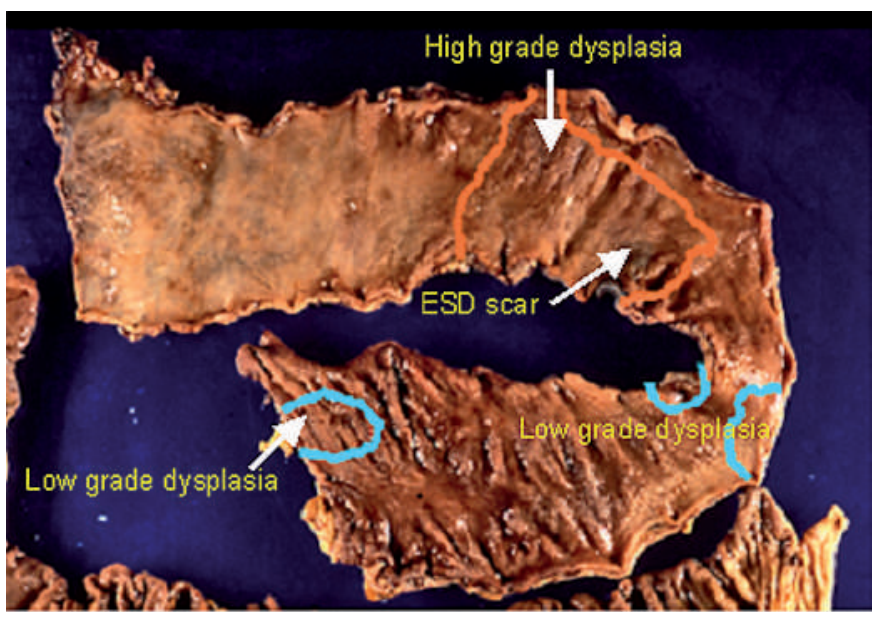

Figure 5 Macroscopic examination of the resected specimen revealed inactive ulcerative colitis with mucosal atrophy, with no evidence of a neoplastic lesion. However, histopathological examination revealed high-grade dysplasia in the mucosa surrounding the post-ESD scar. Histopathological examination of the ascending colon also revealed two areas of low-grade dysplasia.

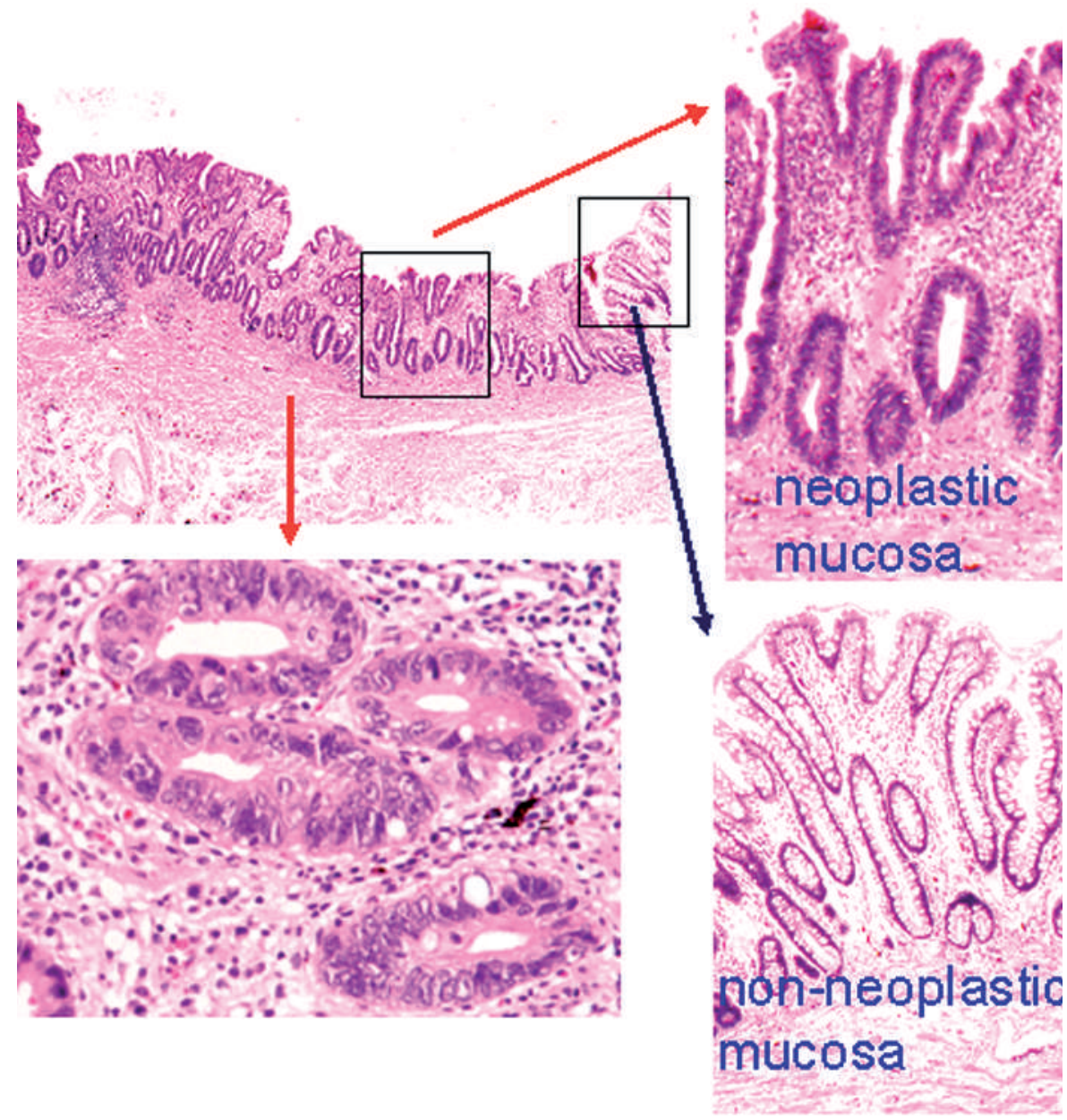

Figure 6 Histopathological examination revealed high-grade dysplasia in the mucosa surrounding the scar after ESD. The structure of the glands, especially the density of the glands of the neoplastic mucosa, resembled that of non-neoplastic colonic mucosa (hematoxylin \& eosin stain). non-neoplastic mucosa (Figure 6). These results suggest that chromoendoscopy, even with pit pattern analysis, has its limitations when used in the diagnosis of intramucosal neoplastic lesions associated with ulcerative colitis.

Endoscopy_UCTN_Code_CCL_1AD_2AC

\section{H. Suzuki'1, Y. Saito', Y. Moriya²,} T. Shimoda ${ }^{3}$, D. Saito ${ }^{1}$

${ }^{1}$ Divison of Endoscopy, National Cancer Center Hospital, Tokyo, Japan

2 Division of Colorectal Surgery, National Cancer Center Hospital, Tokyo, Japan

${ }^{3}$ Clinical Laboratory Division, National Cancer Center Hospital, Tokyo, Japan.

\section{References}

${ }^{1}$ Hata K, Watanabe T, Kazama Set al. Earlier surveillance colonoscopy programme improves survival in patients with ulcerative colitis associated colorectal cancer: results of a 23-year surveillance programme in the Japanese population. Br J Cancer 2003; 89: $1232-1236$

2 Rutter MD, Saunders BP, Schofield Get al. Pancolonic indigo carmine dye spraying for the detection of dysplasia in ulcerative colitis. Gut 2004; 53: 256-260

${ }^{3}$ Kiesslich R, Neurath MF. Surveillance colonoscopy in ulcerative colitis: magnifying chromoendoscopy in the spotlight. Gut 2004; 53: 165 - 167

${ }^{4}$ Fujii T, Hasegawa RT, Saito Yet al. Chromoscopy during colonoscopy. Endoscopy 2001; 33: $1036-1041$

${ }^{5}$ Saito Y, Emura F, Matsuda Tet al. A new sinker-assisted endoscopic submucosal dissection for colorectal cancer. Gastrointest Endosc 2005; 62: 297-301

\section{Corresponding Author}

\section{Y. Saito, M.D.}

Division of Endoscopy

National Cancer Center Hospital

5-1-1 Tsukiji

Chuo-ku

Tokyo 104-0045

Japan

Fax: $\quad+81-3-3542-3815$

E-mail: harusuzu@ncc.go.jp 